\title{
Mixed multiscale finite element methods for stochastic porous media flows
}

\author{
J. E. Aarnes* $\quad$ Y. Efendiev ${ }^{\dagger}$
}

August 24, 2007

\begin{abstract}
In this paper, we propose a stochastic mixed multiscale finite element method. The proposed method solves the stochastic porous media flow equation on the coarse grid using a set of pre-computed basis functions. The pre-computed basis functions are constructed based on selected realizations of the stochastic permeability field, and furthermore the solution is projected onto the finite dimensional space spanned by these basis functions. We employ multiscale methods using limited global information since the permeability fields do not have apparent scale separation. The proposed approach does not require any interpolation in stochastic space, and can easily be coupled with interpolation based approaches to predict the solution on the coarse grid. Numerical results are presented for permeability fields with Gaussian and exponential variograms.
\end{abstract}

\section{Introduction}

It is often difficult to solve multiscale stochastic porous media equations because of the presence of uncertainties and multiple scales. For this reason, some type of upscaling or coarsening is performed to solve these equations on the coarse grid. However, most of upscaling methods studied in the literature are realization based, i.e., upscaling is performed for an individual realization of stochastic permeability field. Because of large uncertainties that are often present in subsurface properties, the use of realization based coarse-scale models may not be sufficient for fast simulation purposes and uncertainty quantification. In this paper, we propose multiscale approaches which resolve both uncertainties and subgrid scales. These approaches are inexpensive and can be used to perform uncertainty quantification in subsurface applications.

In this paper we employ a mixed multiscale finite element method (MsFEM) for resolving the spatial scales. The main idea of MsFEMs is to incorporate

*SINTEF Applied Mathematics, N-0314 Oslo, Norway. email: Jorg.Aarnes@sintef.no

${ }^{\dagger}$ Department of Mathematics, Texas A\&M University, College Station, TX 77843-3368. email: efendiev@math.tamu.edu. Corresponding author 
the small scale information into finite element basis functions and couple them through a global formulation of the problem (see [22]). The multiscale method in [22] shares some similarities with a number of multiscale numerical methods, such as residual free bubbles [10], variational multiscale method [23], two-scale conservative subgrid approaches [6], and multiscale mortar methods [7]. We remark that special basis functions in finite element methods have been used earlier in $[9,8]$. The multiscale finite element methodology has been modified and successfully applied to two-phase flow simulations in $[24,13,1]$ and extended to nonlinear partial differential equations [19].

The main idea of the proposed approach is to construct multiscale basis functions that, in addition to capturing the small scale information, resolves the spatial variability across realizations in stochastic porous media. This is done by selecting a small family of realizations that are sufficiently scattered in stochastic space, and then compute a set of multiscale basis functions for each realization. Once the basis functions are constructed, the solution of the equation for the Darcy velocity is projected onto the finite dimensional space spanned by the multiscale basis functions. The resulting method can be regarded as an extension of the mixed MsFEM to stochastic porous media equations.

The permeability fields under consideration do not have scale separation. We therefore employ multiscale methods using limited global information in our simulations. These approaches extract information (that is difficult to capture with local approaches) from some precomputed fields that contain non-local information. The use of global information in coarsening is not new and has been employed in upscaling (e.g., [12]) as well as multiscale methods (e.g., [1, 18]).

We note that our approach can also use basis functions computed from local problems without involving any global information. This is especially important when there is scale separation. Moreover, the proposed approach can be applied to simulations on unstructured grids $[4,5]$, and does not, unlike upscaling based approaches, require any interpolation in stochastic space. But, if desirable, the method can employ, or be combined with, interpolation based approaches, e.g. by interpolating in stochastic space to construct basis functions.

We present numerical results for two types of permeability fields. The first type is Gaussian permeability field where the uncertainties can be represented by a small number of parameters obtained from Karhunen-Loève expansion [25, 31]. This allows us to select realizations based on sparse interpolation methods and use these realizations to construct multiscale basis functions. The second type of permeability fields are described by the exponential variogram [14]. In this case, we deal with a large uncertainty space and interpolation in uncertainty space is prohibitively expensive. Since our approaches are independent of interpolation, we suggest using a few independent realizations for approximating statistical properties of the stochastic solution. Our results show that the proposed approaches work well and can be used in practice to perform uncertainty quantification, e.g., within the framework introduced in [20].

The paper is organized as follows. In the next section, we present the model problem. Section 3 is devoted to mixed multiscale finite element methods. In the following section, we present mixed multiscale methods for stochastic flow 
equations. Finally, Section 5 is devoted to numerical results.

\section{Model problem}

We consider two-phase flows in a reservoir (denoted by $\Omega$ ) under the assumption that the displacement is dominated by viscous effects; i.e., we neglect the effects of gravity, compressibility, and capillary pressure. The two phases will be referred to as water and oil, designated by subscripts $w$ and $o$, respectively. We write Darcy's law for each phase as follows:

$$
v_{j}=-\frac{k_{r j}(S)}{\mu_{j}} \boldsymbol{k} \cdot \nabla p,
$$

where $v_{j}$ is the phase velocity, $\boldsymbol{k}$ is the permeability tensor, $k_{r j}$ is the relative permeability to phase $j(j=o, w), S$ is the water saturation (volume fraction) and $p$ is pressure. Throughout the paper, we will assume that the permeability tensor is diagonal $\boldsymbol{k}=k \boldsymbol{I}$, where $k$ is a scalar and $\boldsymbol{I}$ is the unit tensor. In this work, a single set of relative permeability curves is used. Combining Darcy's law with a statement of conservation of mass allows us to express the governing equations in terms of the so-called pressure and saturation equations:

$$
\begin{gathered}
\nabla \cdot(\lambda(S) k \nabla p)=q_{w}+q_{o}, \\
\phi \frac{\partial S}{\partial t}+v \cdot \nabla f(S)=q_{w},
\end{gathered}
$$

where $\lambda$ is the total mobility, $f(S)$ is the flux function, and $v$ is the total velocity, which are respectively given by:

$$
\begin{gathered}
\lambda(S)=\frac{k_{r w}(S)}{\mu_{w}}+\frac{k_{r o}(S)}{\mu_{o}}, \\
f(S)=\frac{k_{r w}(S)}{\mu_{w} \lambda(S)}, \\
v=v_{w}+v_{o}=-\lambda(S) k \cdot \nabla p .
\end{gathered}
$$

Moreover, $q_{w}$ and $q_{o}$ are volumetric source terms for water and oil, respectively, and $\phi$ is the porosity. The above descriptions are referred to as the fine model of the two-phase flow problem. For single-phase flow, $k_{r w}(S)=S, k_{r o}(S)=1-S$, and $\mu_{w}=\mu_{o}$.

\section{Mixed multiscale finite element methods}

In this section, we discuss mixed multiscale finite element methods (MsFEM) which will be used in the paper. We rewrite the two-phase flow equation as

$$
\begin{aligned}
(\lambda k)^{-1} u-\nabla p & =0 \quad \text { in } \quad \Omega \\
\operatorname{div}(u) & =h \quad \text { in } \quad \Omega \\
\lambda(x) k(x) \nabla p \cdot n & =g(x) \quad \text { on } \partial \Omega .
\end{aligned}
$$


For simplicity of the presentation, we assume Neumann boundary conditions.

Let $V_{h} \subset H(\operatorname{div}, \Omega)$ and $Q_{h} \subset L^{2}(\Omega) / R$ be finite dimensional spaces and $V_{h}^{0}=V_{h} \cap H_{0}(\operatorname{div}, \Omega)$, where $H_{0}(\operatorname{div}, \Omega)$ is $H(\operatorname{div}, \Omega)$ with homogeneous boundary conditions. The numerical approximation of (3.1) on the fine grid is to find $\left(u_{h}, p_{h}\right) \in V_{h} \times Q_{h}$ such that $u_{h} \cdot n=g_{h}$ on $\partial \Omega$ and

$$
\begin{aligned}
& \left((\lambda k)^{-1} u_{h}, v_{h}\right)+\left(d i v v_{h}, p_{h}\right)=0 \quad \forall v_{h} \in V_{h}^{0} \\
& \left(\text { divu }_{h}, q_{h}\right)=\left(h, q_{h}\right) \quad \forall q_{h} \in Q_{h},
\end{aligned}
$$

where $(\cdot, \cdot)$ is the usual $L^{2}$ inner product.

In a mixed FEM discretization we replace the pressure and velocity solutions by finite dimensional subspaces that typically consists of low order piecewise polynomials. In a mixed MsFEM one attempts to design the approximation space for velocity in such a way that it embodies the impact of subgrid variations in $K$. In a mixed MsFEM, multiscale basis functions are used for the velocity field, while the pressure field is approximated using piecewise constant functions. In particular, one constructs multiscale basis functions for the velocity field for each edge (face in 3 dimensions) of every block. Throughout we denote by $e_{i}^{K}$ an edge or a face $i$ of the block $K$, i.e., a subset of $\partial K$ that has positive length in 2 dimensional space and positive area in 3 dimensional space.

Given a permeability field $k$, the corresponding multiscale basis function for $e_{i}^{K}$ is defined as follows. First we compute an auxiliary function $w_{i}^{K}$ by solving the following equation in $K$ :

$$
\begin{aligned}
\operatorname{div}\left(k(x) \nabla w_{i}^{K}\right) & =\frac{1}{|K|} \quad \text { in } K \\
k(x) \nabla w_{i}^{K} n^{K} & = \begin{cases}g_{i}^{K} & \text { on } e_{i}^{K} \\
0 & \text { else, }\end{cases}
\end{aligned}
$$

where the choice of $g_{i}^{K}$ will be discussed later. To each auxiliary function $w_{i}^{K}$ we associate the multiscale basis function $\Psi_{i}^{K}=k(x) \nabla w_{i}^{K}$. When basis functions have been computed for each edge we define the corresponding finite dimensional velocity approximation space for the mixed MsFEM by

$$
\begin{aligned}
V_{h}(k) & =\bigoplus_{K}\left\{\Psi_{i}^{K}\right\}, \\
V_{h}^{0}(k) & =V_{h}(k) \cap H_{0}(\operatorname{div}, \Omega) .
\end{aligned}
$$

Here we have emphasized that the velocity approximation space depends on $k$, since $k$ appears in the equations that determine the multiscale basis functions. Hence, for each permeability field $k$ we associate a velocity approximation space $V_{h}(k)$ that is spanned by the set of basis functions consisting of one multiscale basis function for each edge of every grid block in the coarse grid. In this paper we employ an approximation space that is defined for multiple $k$, i.e., we use

$$
V_{h}=\bigoplus_{i} V_{h}\left(k_{i}\right),
$$

where $k_{i}(x)$ are permeability fields. This approach, which is useful when there are uncertainties in the permeability data, will be discussed in Section 4 . 


\subsection{Boundary conditions for the multiscale basis functions}

The choice of boundary conditions $g_{i}^{K}$ in (3.3) can greatly influences the accuracy of a mixed MsFEM. In [13], the authors use piecewise constant coarse-scale fluxes on the boundary of the coarse elements, i.e., $g_{i}^{K}=\frac{1}{\left|e_{i}^{K}\right|}$. To obtain accurate approximations of the fluxes, the velocity should contain the fine-scale features similar to the solution of the original problem on the boundary. Piecewise constant boundary conditions can introduce a strong mismatch along grid block boundaries between the mixed MsFEM solution and the solution of the original problem. To alleviate this problem, oversampling method is introduced in [13] (see also $[22,21]$ ). The main idea of the oversampling methods is to use larger regions for local problem computations, i.e., the local problems are solved in larger regions and then the basis functions are computed using only the interior information. These approaches tend to be conservative on the coarse grid only. For our purposes, we would like approaches that are conservative also on the fine grid.

Although the mixed MsFEM is generally robust (see e.g., [4]), it is known that for problems without scale separation and strong non-local effects (e.g., highly heterogeneous fields with long correlation length structures) one can improve accuracy substantially by invoking some type of limited global information $[1,2]$. Global information allows us to take into account strong non-local effects and obtain accurate approximation on the coarse grid. For global approaches to be effective, one needs to know that the solution smoothly depends on these global fields, for example, there exist $N$ global fields $p_{1}, \ldots, p_{N}$, such that

$$
\left|p-G\left(p_{1}, \ldots, p_{N}\right)\right|_{1, \Omega} \leq C \delta,
$$

where $\delta$ is sufficiently small, $G$ is sufficiently smooth function (e.g., $G \in C^{2}$ ), and $p_{1}, . ., p_{N}$ are solutions of $\operatorname{div}(k(x) \nabla p)=0$ with some prescribed boundary conditions. Next, we briefly discuss the assumptions such as (3.4) and then state mixed MsFEM which use limited global information. The latter will be used in our simulations.

In [18], it was shown for channelized permeability fields that $p$ is a smooth function of single-phase flow pressure (i.e., $N=1$ ), where single-phase pressure equation is described by $\operatorname{div}(k(x) \nabla p)=f$. In more general setting, it was shown in [28] for arbitrary smooth $\lambda(x)$ that the solution is a smooth function of two linearly independent solutions of single-phase flow equations $(N=2)$. When considering random permeability fields, the permeability field is typically parameterized with a parameter that represents the uncertainties and in this case the global fields are the solutions of single-phase flow equation for different realizations. This will be discussed in next section.

In the above assumption, $p_{i}$ are solutions of flow equations. We denote the corresponding velocity field by $u_{i}$, i.e., $u_{i}=k \nabla p_{i}$. Then, the above assumption can be written in the following way. There exist sufficiently smooth scalar functions $A_{1}(x), \ldots, A_{N}(x)$ (see [3] for details), such that the velocity can be written as

$$
\left\|u-A_{1}(x) u_{1}-\ldots-A_{N}(x) u_{N}\right\|_{0, \Omega} \leq C \delta .
$$


We note that in the case of homogenization problems $u_{i}=k(x) \nabla p_{i}$, where $p_{i}$ are solutions of local periodic problems such that $p_{i}-x_{i}$ is periodic $(i=1, \ldots, d)$.

The idea of using global information to define boundary conditions for the multiscale basis functions for the mixed MsFEM was first introduced in [1] (for $N=1$ ). Here we define a mixed MsFEM capable of using information from multiple global fields. This approach is therefore a generalization of the mixed MsFEM proposed in [1].

\section{Mixed MsFEM for stochastic equations}

In this section, we present a multiscale approach for solving stochastic flow equations. The main idea is to use selected realizations of the permeability field to build a low dimensional approximation space for velocity that embodies both small scale (sub coarse-grid) spatial variability in the permeability data and stochastic variability due to uncertainties in the data. This space can then be utilized to compute velocity solutions for other realizations from the stochastic permeability distribution. For clarity we summarize the basic steps below.

1. Generation of coarse grid.

- Let the reservoir $\Omega$ be partitioned into a fine grid. The coarse grid is a partitioning of the fine grid where each cell in the fine grid belongs to a unique block in the coarse grid and each coarse grid block is connected. In principle, any grid satisfying these requirements may be used, but a proper coarse grid should obey certain guidelines, see [5].

2. Construction of multiscale approximation space $V_{h}$ :

- Select $N$ realizations from the stochastic permeability distribution that are scattered in stochastic space.

- For each realization $i$;

- Solve (3.1) on the fine grid using a suitable mass conservative numerical method to obtain a "global" velocity solution $u_{i}$.

- Compute multiscale basis functions: For each edge $e_{l}^{K}$, set

$$
g_{l}^{K}\left(k_{i}\right)=\frac{u_{i} \cdot n_{e_{l}}^{K}}{\int_{e_{l}} u_{i} \cdot n_{e_{l}}^{K} d s}
$$

where $n_{e_{l}}^{K}$ is the unit normal on $e_{l}$ pointing out of $K$, and solve (3.3) to obtain $w_{l}^{K}$ and subsequently $\Psi_{l}^{k}$.

- Define $V_{h}\left(k_{i}\right)=\bigoplus_{l, K} \Psi_{l}^{K}$.

- Define $V_{h}=\bigoplus_{i=1}^{N} V_{h}\left(k_{i}\right)$. 
3. Rapid multiscale computation of velocity solutions for stochastic porous media flow with precomputed basis functions (from Step 2) for each individual realization, e.g., for uncertainty quantification, history matching, etc.

- Select a family of realizations.

- For each realization, solve (3.2) with $V_{h}^{0}=V_{h} \cap H_{0}(\operatorname{div}, \Omega)$.

We see that using the above procedure we get $N$ basis functions for each edge in the coarse grid. Once the basis functions are constructed, the mixed MsFEM computes the orthogonal projection of the corresponding "global" velocity solution onto $V^{h}$ with respect to the inner-product $\left(\cdot(\lambda k)^{-1}, \cdot\right)$. Hence, the mixed MsFEM projects the "true" solution of the flow equation for any realization onto the approximation space $V_{h}$ that was built a priori.

We note that the mixed MsFEM finds a proper solution for each realization without using interpolation formulas. However, for the mixed MsFEM solution to be well defined it is necessary that all basis functions for all of the initial $N$ realizations are linearly independent. This is the case if each family of boundary conditions $\left\{g_{l}^{K}\left(k_{i}\right):\right.$ all $\left.i, K\right\}$ is a linear independent set. If this is not the case, then one must remove superfluous boundary conditions and let $V_{h}$ be spanned by the basis functions that correspond to the reduced set of boundary conditions. In practice this is usually not necessary as long as the number of realizations used in constructing multiscale basis functions is not too high.

\subsection{Analysis and discussion}

Next, we present a formal analysis of the method under the assumption that the chosen realizations can be used to interpolate an arbitrary realization. To show this, we assume that the uncertainties of the permeability field can be parameterized. As a result of this parameterization, the permeability is expressed as $k=k(x, \theta)$ where $\theta \in R^{L}$. One such example is the KarhunenLoève expansion (KLE) used in representing the permeability fields given via a two-point correlation function. In the KLE, the permeability is expressed as $k(x, \theta)=\exp (Y(x, \theta))$ with $Y(x, \theta)=\sum_{i=1}^{L} \Theta_{i} \Phi_{i}(x)$. Here $\Phi_{i}(x)$ are predetermined functions and $\theta=\left(\Theta_{1}, \ldots, \Theta_{L}\right)$. The KLE is further described in the section on numerical implementation.

When the uncertainties are parameterized and $L$ is not large, one can employ sparse interpolation techniques in $R^{L}$ (e.g., [32]), where the solution is computed for some values of $\theta=\left(\Theta_{1}, \ldots, \Theta_{L}\right)$, denoted by $\theta_{k}$, and then interpolated for an arbitrary $\theta \in R^{L}$. Assuming that $k(x, \theta)$ smoothly depends on $\theta$ (keeping in mind Karhunen-Loève type expansions), we can approximate the solution for an arbitrary $\theta$ as

$$
p(x, \theta) \approx \sum_{i} p\left(x, \theta_{i}\right) \beta_{i}(\theta),
$$

where $\beta_{i}(\theta)$ are the corresponding weights which are in general difficult to obtain. We note that the interpolation error depends on the choice of interpolation 
points and the smoothness of $p(x, \theta)$ with respect to $\theta$. Denoting the velocity field for two-phase flow by $v$, we have

$$
v(x, \theta) \approx \sum_{i} v\left(x, \theta_{i}\right) \beta_{i}(\theta) .
$$

(4.1) shows that the solution of stochastic flow equation can be approximated if we provide approximations of $p\left(x, \theta_{i}\right)$ for each $\theta_{i}$. Because the solution for each selected realization can be approximated using corresponding global fields, we have

$$
v\left(x, \theta_{i}\right) \approx \sum_{j} c_{i j}^{*}(x) u_{j}\left(x, \theta_{i}\right) .
$$

We remind that $j$ refers to a number of global fields used for simulations of a single realization. In our numerical simulations, we will be using single-phase velocity fields following $[1,2]$. One can, in general, use directional flows as it is proposed in more general setting in [28]. We note that in our multiscale simulations, the basis functions are constructed using $u_{j}\left(x, \theta_{i}\right)$.

One can show the convergence of the proposed approach following [3]. From (4.1) and (4.2), we obtain that

$$
v(x, \theta) \approx \sum_{i} \beta_{i}(\theta) \sum_{j} c_{i j}^{*}(x) u_{j}\left(x, \theta_{i}\right) .
$$

This shows that the solution can be approximated by $u_{j}\left(x, \theta_{i}\right)$. More precisely, we have

$$
\left\|v(x, \theta)-\sum_{i, j} \beta_{i}(\theta) c_{i j}^{*}(x) u_{j}\left(x, \theta_{i}\right)\right\|_{L^{2}(\Omega)} \leq \delta,
$$

where $\delta$ is a sufficiently small number and $\Omega$ is the spatial domain. $\delta$ in (4.4) depends on $\theta$ (realization). Following the proof presented in [3] one can show that the mixed multiscale finite element methods converge independent of small scales and, in particular, the convergence rate is $C\left(\delta+h^{\gamma}\right)$, where $C$ depends on $\theta, h$ is the coarse mesh size, and $0<\gamma<1$ depends on the smoothness of $A_{i}$ in (3.5). We will not present this proof here since it is lengthy. For the proof, one needs first to establish a stability estimate and then obtain the convergence rate. Because basis functions span $u_{j}\left(x, \theta_{i}\right)$, one can appropriately choose a test function which will yield the desired convergence rate. Once the convergence for each $\theta$ is obtained, we can show the uniform convergence in a compact set in $R^{L}$ using Ascoli theorem.

We note that the proposed method can be applied in a local region of the uncertainty space by selecting realizations which correspond to this region. The latter is useful when one would like to perform uncertainty quantification in a subregion of the uncertainty space. One can also use the localization in the uncertainty space for more accurate probabilistic estimations by partitioning the uncertainty space. To describe the procedure, we denote by $U$ the uncertainty space and assume that $U$ is partitioned into $U_{i}$. In each region $U_{i}$, we choose realizations $\theta_{j}^{i}$ representing these local regions. Then, the basis functions are 
defined as before for these realizations in each $U_{i}$. This approach is an implementation of the earlier proposed technique simply in local regions of uncertainty space. In particular, the multiscale basis functions are constructed as before though with local support both in spatial and uncertainty spaces. When performing simulations for a particular realization, the multiscale basis functions from the local uncertainty region which contains this particular realization will be used. This will provide high accuracy and reduce the computational cost. We note that pre-computed multiscale basis functions can be repeatedly used for different boundary conditions/source terms and for dynamic two-phase flow and transport simulations.

One can draw a parallel between the proposed approach and a general multiscale approach where the coefficients strongly vary with respect to spatial variables and uncertainties. In classical multiscale finite element type methods, the local spatial heterogeneities are captured via the solution of the local equation in the spatial space. We would like to construct multiscale basis functions for permeability fields $k(x, \theta)$ over a coarse region defined in the spatial and uncertainty space (we denote it $K \times U_{i}$ ). Analogous to the spatial multiscale methods, here we attempt to solve the local problem in a coarse region $K \times U_{i}$. More precisely, the basis functions are derived from the solution of

$$
\operatorname{div}\left(k(x, \theta) \nabla w_{i}^{K}(x, \theta)\right)=0
$$

defined in $K \times U_{i}$. To solve this local problem, a discretization in the spatial and uncertainty space is required. In the proposed stochastic mixed MsFEM, besides a standard finite element based spatial discretization, we use a number of realizations to resolve the uncertainty scales (similar to stochastic collocation methods $[32,26])$. Consequently, the proposed approach can be regarded, conceptually, as an extension of mixed MsFEM to problems with uncertainties.

The main practical advantage of the proposed mixed MsFEM is that one does not need interpolation formulas. Indeed, when an approximation space that is spanned by the family of spaces for a set of different permeability realizations is applied to solve (2.2) with an independent realization of the permeability field, one is actually projecting the true solution onto this enriched approximation space. Thus, the velocity solution will be a superposition of basis functions corresponding to each of the sample fields, but the interpolation weights are determined automatically from the projection property of the mixed MsFEM. In particular, the interpolation weights will vary throughout the uncertainty domain. This approach is interpolation free, easy to use, and provides a computationally cost-efficient methodology for performing multiple simulations, for instance to quantify uncertainty. We also note that when an interpolation formula is easily available, one can interpolate the set of pre-computed multiscale basis functions to calculate the basis functions for a particular realization. However, the nature of this interpolation (pointwise or $L^{2}$ or etc) will be predetermined. Our proposed approach chooses the best interpolation both in spatial and stochastic space.

To our best knowledge, one can not avoid interpolation techniques if an upscaled model for an arbitrary realization is sought. Indeed, to derive coarse 
grid models with prescribed grid block porosities and permeability tensors for multiple realizations, one must either upscale each individual realization separately, or upscale a selected subset of the realizations and employ interpolation formulas to extrapolate coarse grid models for the un-upscaled realizations (see [15]). The former approach is time demanding because the upscaling part is often time-consuming relative to the time it takes to perform a coarse grid simulation. The latter approach, on the other hand, is limited to problems where interpolation formulas are readily available. We should remark, however, that approaches proposed in [11] can be used to derive an ensemble level information for upscaled permeabilities without employing interpolation.

\section{$5 \quad$ Numerical results}

For our numerical tests, we use the Karhunen-Loève expansion (KLE) [25, 31] to obtain the permeability field in terms of an optimal $L^{2}$ basis. By truncating

the expansion, we can represent the permeability matrix by a small number of random parameters. To impose the hard constraints (the values of the permeability at prescribed locations), one can find a linear subspace of our parameter space (a hyperplane) which yields the corresponding values of the permeability field. First, we briefly recall the facts of the KLE. Denote $Y(x, \omega)=\log [k(x, \omega)]$, where the random element $\omega$ is included to remind us that $k$ is a random field. For simplicity, we assume that $E[Y(x, \omega)]=0$. Suppose $Y(x, \omega)$ is a second order stochastic process with $E \int_{\Omega} Y^{2}(x, \omega) d x<\infty$, where $E$ is the expectation operator. Given an orthonormal basis $\left\{\phi_{k}\right\}$ in $L^{2}(\Omega)$, we can expand $Y(x, \omega)$ as

$$
Y(x, \omega)=\sum_{k=1}^{\infty} Y_{k}(\omega) \phi_{k}(x), \quad Y_{k}(\omega)=\int_{\Omega} Y(x, \omega) \phi_{k}(x) d x .
$$

We are interested in the special $L^{2}$ basis $\left\{\phi_{k}\right\}$ which makes the random variables $Y_{k}$ uncorrelated. That is, $E\left(Y_{i} Y_{j}\right)=0$ for all $i \neq j$. Denote the covariance function of $Y$ as $R(x, y)=E[Y(x) Y(y)]$. Then such basis functions $\left\{\phi_{k}\right\}$ satisfy

$$
E\left[Y_{i} Y_{j}\right]=\int_{\Omega} \phi_{i}(x) d x \int_{\Omega} R(x, y) \phi_{j}(y) d y=0, \quad i \neq j .
$$

Since $\left\{\phi_{k}\right\}$ is a complete basis in $L^{2}(\Omega)$, it follows that $\phi_{k}(x)$ are eigenfunctions of $R(x, y)$ :

$$
\int_{\Omega} R(x, y) \phi_{k}(y) d y=\lambda_{k} \phi_{k}(x), \quad k=1,2, \ldots
$$

where $\lambda_{k}=E\left[Y_{k}^{2}\right]>0$. Furthermore, we have

$$
R(x, y)=\sum_{k=1}^{\infty} \lambda_{k} \phi_{k}(x) \phi_{k}(y)
$$


Denote $\theta_{k}=Y_{k} / \sqrt{\lambda_{k}}$, then $\theta_{k}$ satisfy $E\left(\theta_{k}\right)=0$ and $E\left(\theta_{i} \theta_{j}\right)=\delta_{i j}$. It follows that

$$
Y(x, \omega)=\sum_{k=1}^{\infty} \sqrt{\lambda_{k}} \theta_{k}(\omega) \phi_{k}(x),
$$

where $\phi_{k}$ and $\lambda_{k}$ satisfy (5.1). We assume that the eigenvalues $\lambda_{k}$ are ordered as $\lambda_{1} \geq \lambda_{2} \geq \ldots$. The expansion (5.3) is called the Karhunen-Loève expansion. In the KLE (5.3), the $L^{2}$ basis functions $\phi_{k}(x)$ are deterministic and resolve the spatial dependence of the permeability field. The randomness is represented by the scalar random variables $\theta_{k}$. After we discretize the domain $\Omega$ by a rectangular mesh, the continuous KLE (5.3) is reduced to finite terms. Generally, we only need to keep the leading order terms (quantified by the magnitude of $\lambda_{k}$ ) and still capture most of the energy of the stochastic process $Y(x, \omega)$. For an $N$-term KLE approximation $Y_{N}=\sum_{k=1}^{N} \sqrt{\lambda_{k}} \theta_{k} \phi_{k}$, define the energy ratio of the approximation as

$$
e(N):=\frac{E\left\|Y_{N}\right\|^{2}}{E\|Y\|^{2}}=\frac{\sum_{k=1}^{N} \lambda_{k}}{\sum_{k=1}^{\infty} \lambda_{k}}
$$

If $\lambda_{k}, k=1,2, \ldots$, decay very fast, then the truncated KLE would be a good approximation of the stochastic process in the $L^{2}$ sense.

Suppose the permeability field $k(x, \omega)$ is a log-normal homogeneous stochastic process, then $Y(x, \omega)$ is a Gaussian process, and $\theta_{k}$ are independent standard Gaussian random variables. In this case, the covariance function of $Y(x, \omega)$ has the form

$$
R(x, y)=\sigma^{2} \exp \left(-\frac{\left|x_{1}-y_{1}\right|^{2}}{2 l_{1}^{2}}-\frac{\left|x_{2}-y_{2}\right|^{2}}{2 l_{2}^{2}}\right) .
$$

In the above formula, $l_{1}$ and $l_{2}$ are the correlation lengths in each dimension, and $\sigma^{2}=E\left(Y^{2}\right)$ is a constant. We first solve the eigenvalue problem (5.1) numerically on the rectangular mesh and obtain the eigenpairs $\left\{\lambda_{k}, \phi_{k}\right\}$. Since the eigenvalues decay fast, the truncated KLE approximates the stochastic process $Y(x, \omega)$ fairly well in the $L^{2}$ sense. Therefore, we can sample $Y(x, \omega)$ from the truncated KLE (5.3) by generating Gaussian random variables $\theta_{k}$.

\subsection{Experimental setup}

In our simulations below, we take $k_{r w}(S)=S^{2}, \mu_{w}=0.1, k_{r o}(S)=(1-S)^{2}$, and $\mu_{o}=1$. The permeability field $Y(x)$ is given on $100 \times 100$ fine Cartesian grid. This grid is then coarsened to form a uniform $5 \times 5$ Cartesian grid so that each block in the coarse grid contains a $20 \times 20$ cell partition from the fine grid. We solve the pressure equation on the coarse grid using the mixed MsFEM and then reconstruct the fine-scale velocity field as a superposition of the multiscale basis functions. The reconstructed field is used to solve the saturation equation. The saturation equation will be solved using an implicit upstream finite volume (discontinuous Galerkin) method. For emphasis, we note that the multiscale basis functions are constructed at time zero, i.e., they are not recomputed during the simulations. 
In the numerical examples that are reported below we consider a traditional quarter-of-a-five-spot. That is, $\Omega$ is taken to be a square domain, we inject water at the upper left corner, and produce whatever reaches the producer at the lower right corner. To assess the quality of the respective saturation solutions obtained using the mixed MsFEM, we compute for each realization a reference solution $S_{\text {ref }}$ obtained by solving the time-dependent pressure equation on the fine grid with the given permeability field (using the lowest order Raviart-Thomas mixed finite element method for Cartesian grids [29]). Then, in addition to measuring the relative saturation error in the $L^{1}$-norm:

$$
\left\|S-S_{\mathrm{ref}}\right\|_{L^{1}} /\left\|S_{\mathrm{ref}}\right\|_{L^{1}}
$$

we compare various production characteristics. To this end we use the water-cut curve defining the fraction of water in the produced fluid as a function of time measured in pore volumes injected (PVI). Thus,

$$
w(t)=\frac{q_{w}(t)}{q_{w}(t)+q_{o}(t)},
$$

where $q_{o}$ and $q_{w}$ are flow rates of oil and water at the producer at time $t$. When time is measured in PVI we have that $t=\frac{1}{\int_{\Omega} \phi d x} \int_{0}^{t}\left(q_{w}(\tau)+q_{o}(\tau)\right) d \tau$.

Having defined the water-cut, we monitor the following quantities:

- The relative water-cut error in the $L^{2}$-norm:

$$
\left\|w-w_{\text {ref }}\right\|_{L^{2}} /\left\|w_{\text {ref }}\right\|_{L^{2}} .
$$

- The breakthrough time (defined as $w^{-1}(0.05)$ ) at the producer.

- The cumulative oil production at 0.6 PVI:

$$
Q_{o}=-\frac{1}{\int_{\Omega} \phi d x} \int_{0}^{0.6 P V I}\left(\int_{\Omega} \min \left(q_{o}(x, \tau), 0\right) d x\right) d \tau .
$$

Before we embark on the numerical experiments, we note that the RaviartThomas mixed finite element discretization of the pressure equation results in a linear system with $100^{2}+2 \times 99 \times 100=29800$ unknowns. In comparison, when using a sample of $N$ permeability fields to generate the mixed MsFEM basis functions, the stochastic multiscale method gives rise to a linear system with $5^{2}+2 \times 4 \times 5 \times N=25+40 N$ unknowns. Hence, when using a sample size of 25 , for instance, the number of the unknowns in the fine grid system is roughly 30 times larger than the number of unknowns in the mixed MsFEM system. In this paper we will employ samples of 10-50 permeability fields. In other words, we compute 10-50 velocity basis functions for each interface in the coarse grid.

We note that the dimension of the multiscale approximation space may be reduced. For instance, as mentioned earlier, the proposed approach can be used locally in the uncertainty space. This requires partitioning of the uncertainty 
space and for each realization determining the partition it belongs to. Also, when basis functions that correspond to the same interface in the coarse grid are nearly linear dependent, then the number of basis functions may be reduced, e.g., by removing the basis functions that are at a very small angle (with respect to $\left.\left(\cdot(\lambda k)^{-1}, \cdot\right)\right)$ to the space spanned by the remaining basis functions associated with the same interface. These techniques reduce the computation cost without losing essential information.

\subsection{Gaussian fields}

For Gaussian fields, one can reduce the dimension of the uncertainty space dramatically due to the fast decay of eigenvalues. To sample the realizations that are used to generate the multiscale basis functions, we use the 1st order Smolyak collocation points $\theta_{k}$ in $[-3,3]^{L}$ (see e.g., [32]). That is, $\theta_{0}=0, \theta_{2 i-1}=3 \delta_{i j}$, and $\theta_{2 i}=-3 \delta_{i j}, i=1, \ldots, L$. We note that the choice of interpolation points does not affect the implementation of our approach.

Our first results are for isotropic case with $l_{1}=l_{2}=0.2$ and $\sigma^{2}=2$. In this case we can reduce the dimension of the stochastic permeability to 10. From this stochastic model for the permeability we randomly draw 100 realizations and perform simulations on the corresponding permeability fields.

In Figure 1 we compare breakthrough times and cumulative oil production at 0.6 PVI. We see that there is nearly a perfect match between the results obtained with mixed MsFEM and the corresponding results derived from the reference solutions. Next, in Figure 2, we plot $L^{2}$ errors in the saturation field for these realizations as well as the water-cut errors. It can be observed from this figure that the saturation errors are mostly below 3\%. Finally, we plot in Figure 3 a histogram of the breakthrough times and cumulative oil production values depicted in Figure 1 to demonstrate that the mixed MsFEM essentially provides the same statistics as one obtains from the set of reference solutions. These results suggest that with a few pre-computed basis functions in each coarse grid block we can solve two-phase flow equations on the coarse grid for an arbitrary realization and obtain nearly the same results, and hence nearly the same statistics, as one obtains by doing fine grid simulations for each realization.

In our next numerical example, we consider an anisotropic Gaussian field with $l_{1}=0.5, l_{2}=0.1$ and $\sigma^{2}=2$. Due to anisotropy, it requires 12 terms in KLE. Again we sample the realizations that are used to generate the multiscale basis functions using the 1 st order Smolyak collocation points $\theta_{k}$ in $[-3,3]^{L}$. For illustration purposes, we plot in Figure 4 one randomly chosen realization and corresponding saturation profiles at 0.6 PVI obtained by solving the pressure equation on the fine grid, and on the $5 \times 5$ coarse grid with the mixed MsFEM, respectively. We note that the depicted saturation profiles are approximately the same, even though the multiscale method solves the pressure equation on a grid that is coarsened 20 times in each direction.

The numerical results obtained for the anisotropic Gaussian fields are qualitatively the same as the results shown in Figure 1 - Figure 3. We therefore include only the anisotropic equivalent of Figure 3. Histograms of breakthrough 

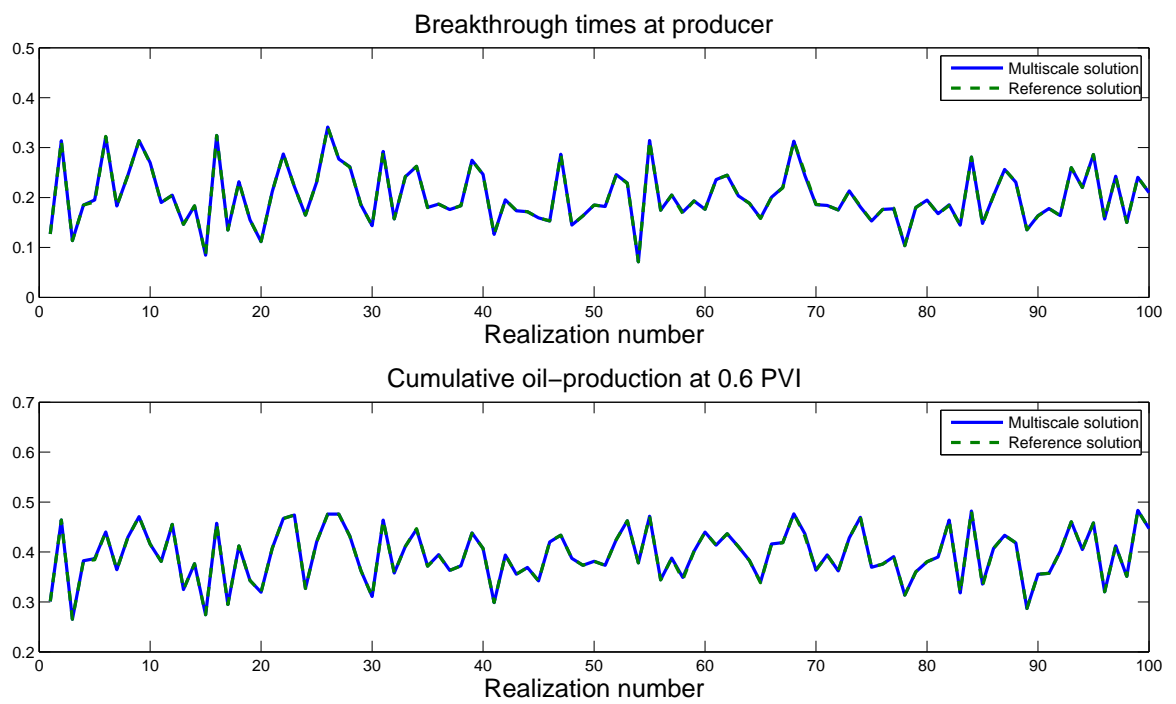

Figure 1: Breakthrough time and cumulative oil production at 0.6 PVI for 100 random realizations from a Gaussian field with $l_{1}=l_{2}=0.2$ and $\sigma^{2}=2$.

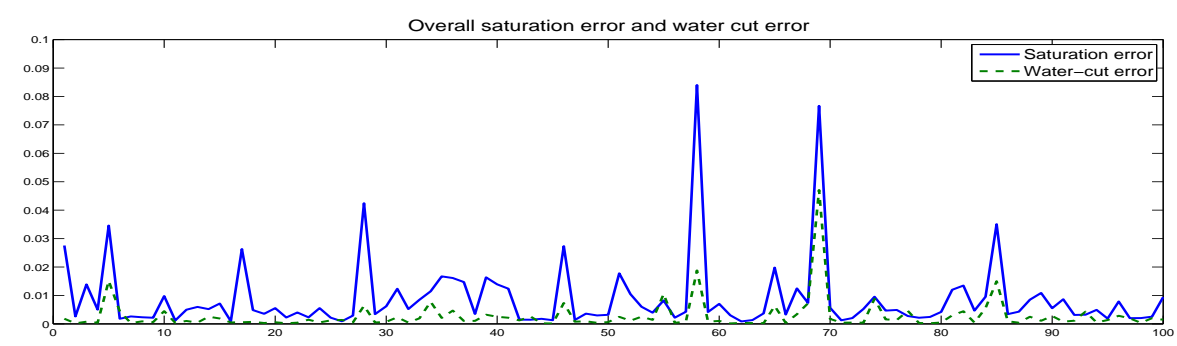

Figure 2: $L^{2}$ errors of the saturation field and water-cut errors for 100 randomly chosen realizations. Gaussian field with $l_{1}=l_{2}=0.2, \sigma^{2}=2$.
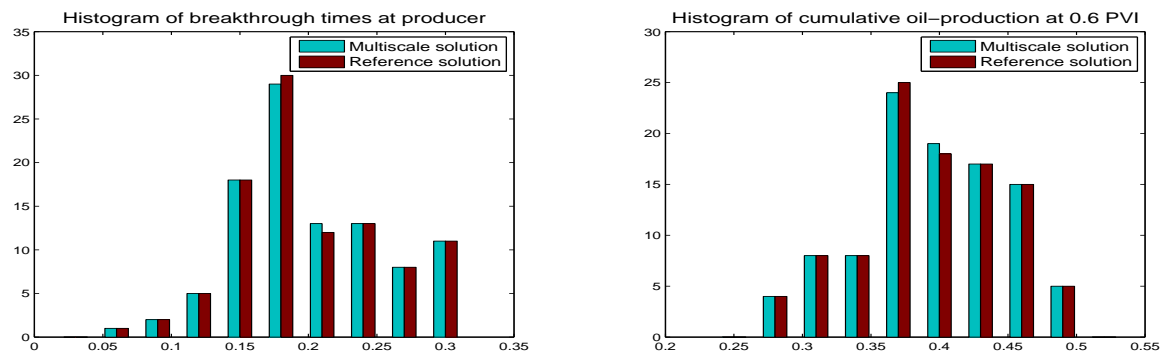

Figure 3: Histograms of the breakthrough times and cumulative oil production values shown in Figure 1. 
time and cumulative oil production at 0.6 PVI for 100 randomly chosen realizations are depicted in Figure 5. The histograms confirm that the multiscale method essentially provides the same breakthrough time and cumulative oil production statistics as one obtains from the set of reference solutions.

\subsection{Exponential variogram fields}

For our second set of results, we consider permeability fields with exponential covariance matrix

$$
R(x, y)=\sigma^{2} \exp \left(-\frac{\left|x_{1}-y_{1}\right|}{l_{1}}-\frac{\left|x_{2}-y_{2}\right|}{l_{2}}\right) .
$$

Because of slow decay of eigenvalues, one usually needs to keep many terms in the KLE and deal with a large uncertainty space. For instance, for approximating permeability fields, KLE may require 300 to 400 eigenvectors depending on correlation lengths and variance. The KLE therefore gives a stochastic distribution in which it is very difficult to derive and employ interpolation formulas. Thus, for this problem our approach based on the mixed MsFEM offers a major advantage relative to interpolation based approaches. In fact, in our approach we can simply select a few independent realizations and use these realizations to build the approximation space $V_{h}$, and then perform statistical studies on a much larger set of realizations. We note that for independent realizations, we do not have interpolation formula easily available. Moreover, the use of independent realizations is quite easy and one can use this technique for more general permeability fields since it only requires independent samples of the permeability field.

To demonstrate the performance of the stochastic multiscale method for these fields, we present results for a case where the permeability fields are drawn from an anisotropic exponential variogram distribution with $l_{1}=0.5, l_{2}=0.1$, and $\sigma^{2}=2$ (the results for the isotropic case are similar, and not reported here). The KLE requires 350 eigenvectors to represent this stochastic permeability distribution. From this distribution we sample 20 independent realizations and use these realizations to generate the multiscale basis functions. Figure 6 displays one randomly chosen realization and corresponding saturation profiles at 0.6 PVI obtained by solving the pressure equation on the fine grid, and on the $5 \times 5$ coarse grid with the mixed MsFEM, respectively.

Figures 7, 8, and 9, show; breakthrough time at producer and cumulative oil production at 0.6 PVI for 100 randomly chosen realizations for both the reference solution and the multiscale solution; relative overall saturation error and water-cut error; histograms of the breakthrough times and cumulative oil production values depicted in Figure 7 . Figure 7 demonstrates that there is generally a good match between the breakthrough time and cumulative oil production curves for the reference and multiscale solutions. However, we now observe that there is a slight bias in the multiscale results, e.g., there is a small time-lag in the breakthrough times for the multiscale method. The bias can also be observed from the histograms in Figure 9, but the magnitude of the bias 

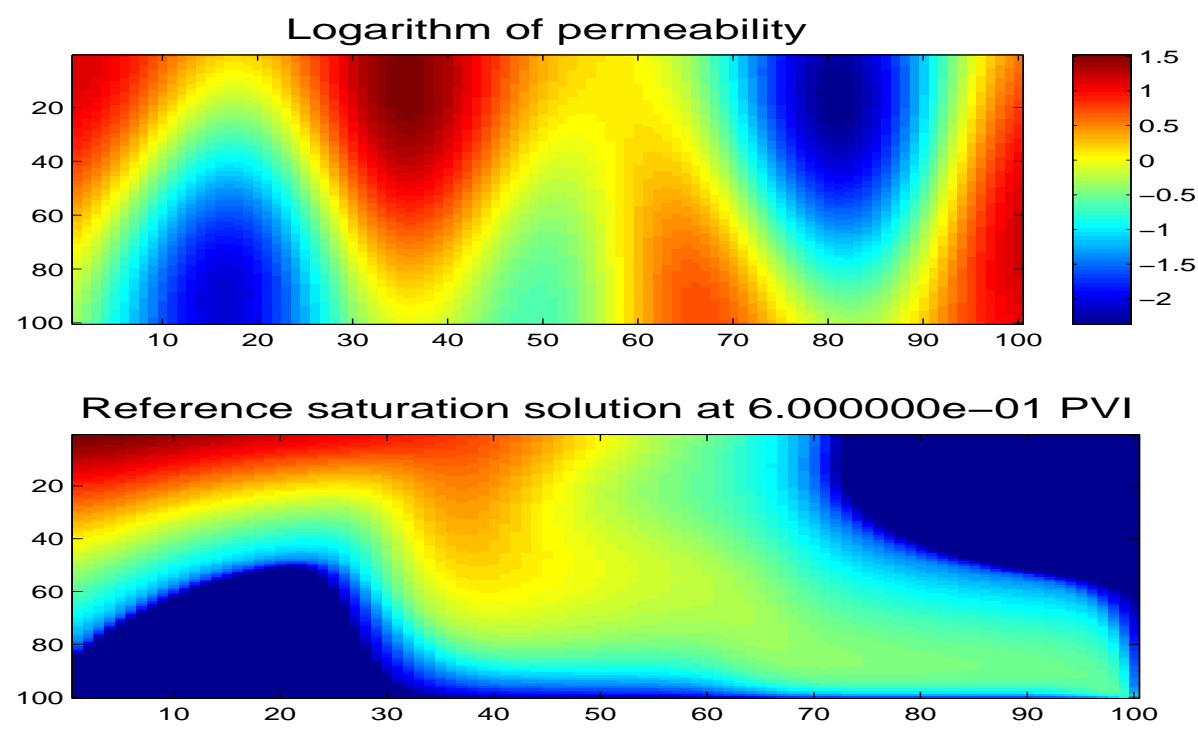

Multiscale saturation solution at $6.000000 e-01 \mathrm{PVI}$

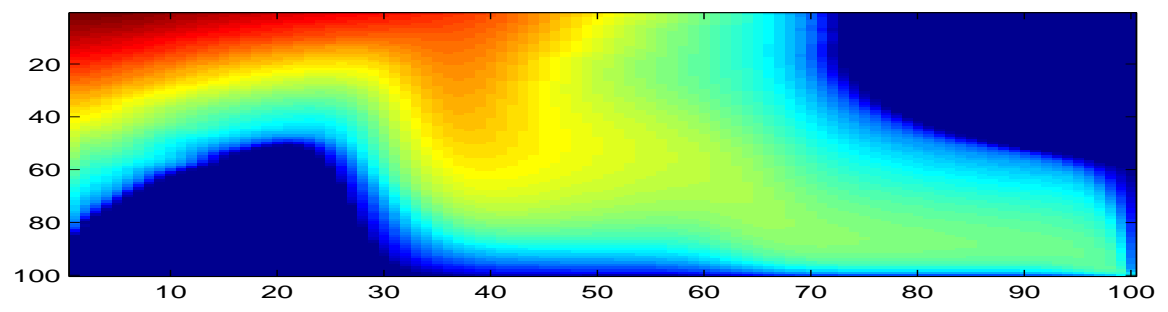

Figure 4: A Gaussian permeability field with $l_{1}=0.5, l_{2}=0.1, \sigma^{2}=2$, and a comparison of the reference saturation field and the saturation profile obtained with the multiscale method at $0.6 \mathrm{PVI}$.
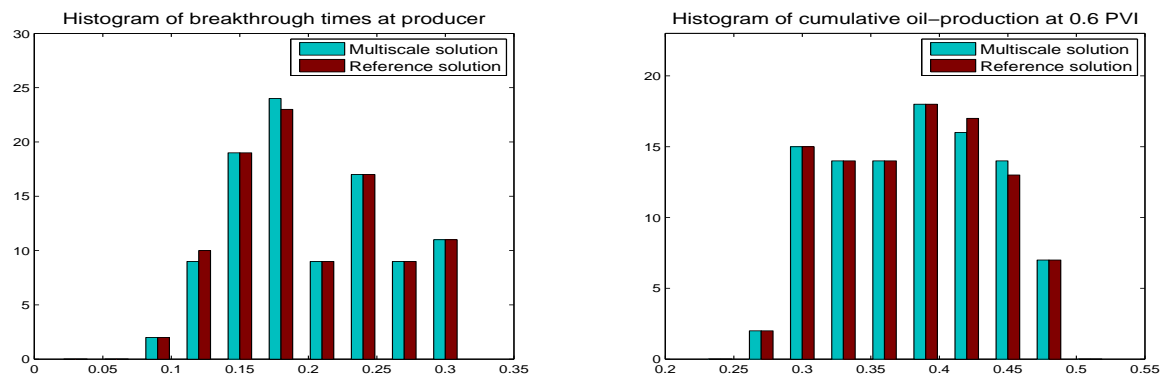

Figure 5: Histograms of breakthrough time and cumulative oil production at 0.6 PVI for 100 random Gaussian fields with $l_{1}=0.5$ and $l_{2}=0.1$ and $\sigma^{2}=2$. 

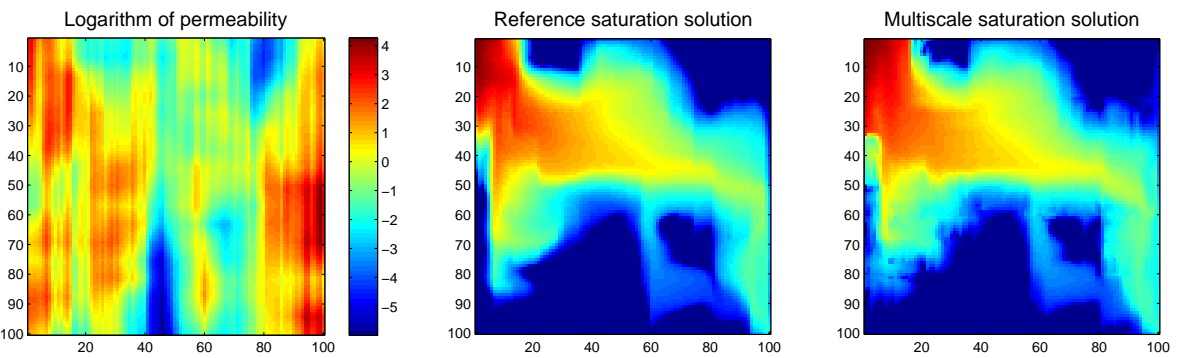

Figure 6: An exponential variogram field with $l_{1}=0.5, l_{2}=0.1$, and $\sigma^{2}=2$, and a comparison of the reference saturation field at $0.6 \mathrm{PVI}$ and the corresponding saturation field obtained using the stochastic multiscale method.

is small, and the multiscale solutions are generally quite close to the reference solution, as is illustrated in Figure 6 and Figure 8.

We now demonstrate that the bias in breakthrough time and cumulative oil production persists, but is efficiently reduced by increasing the number of realizations used to generate the multiscale basis functions. Figures 10, 11, and 12 show, respectively, the saturation and water-cut error for each of the 100 realization for the stochastic multiscale method with different sample sizes, the cumulative probability distribution of breakthrough times and cumulative oil production, and the corresponding histograms of the breakthrough times and the cumulative oil production values. The plots show the following; the saturation and water-cut errors decay with increasing sample size; the time lag in the breakthrough times (also observed in the cumulative oil production) decays rapidly with increasing sample size, and that using 50 basis functions for each coarse grid interface generates statistics that are nearly unbiased, and generally match the statistics derived from the set of reference solutions very well. Observe that a sample size of 50 gives rise to a linear system with 2025 unknowns, roughly $1 / 15$ as many as in the fine grid system.

\subsection{Uncertainty quantification}

We would like to note that the proposed techniques can be effectively used in uncertainty quantification based on methods proposed in [20]. Our goal in [20] is to sample the permeability from the distribution

$$
\pi(k) \propto \exp \left(-\frac{1}{s^{2}} \int_{0}^{T}\left(q_{w}(t)-q^{o b s}(t)\right)^{2} d t\right),
$$

where $q_{w}$ is the water-cut corresponding to the permeability field $k, q^{o b s}$ is observed water-cut, $T$ is the time of available history and $s$ is the error precision.

In [20], multi-stage Markov chain Monte Carlo methods are proposed, where the first stage involves inexpensive simulations. These simulations are used to 

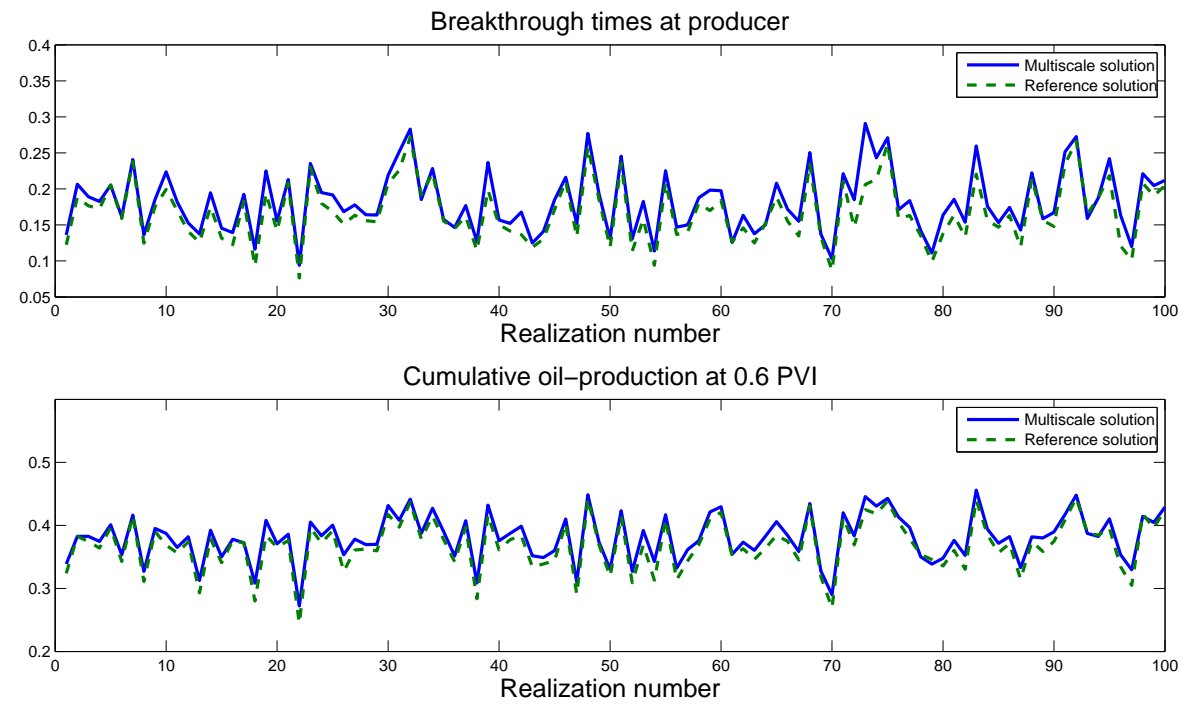

Figure 7: Breakthrough time and cumulative oil production at 0.6 PVI for 100 random realizations from an exponential variogram field with $l_{1}=0.5, l_{2}=0.1$, and $\sigma^{2}=2$.

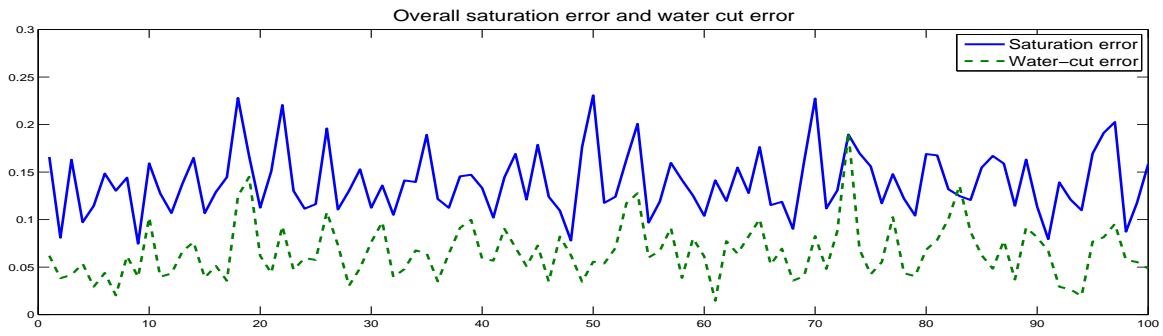

Figure 8: $L^{2}$ errors of the saturation field and water-cut errors for 100 randomly chosen exponential variogram fields with $l_{1}=0.5, l_{2}=0.1$, and $\sigma^{2}=2$.
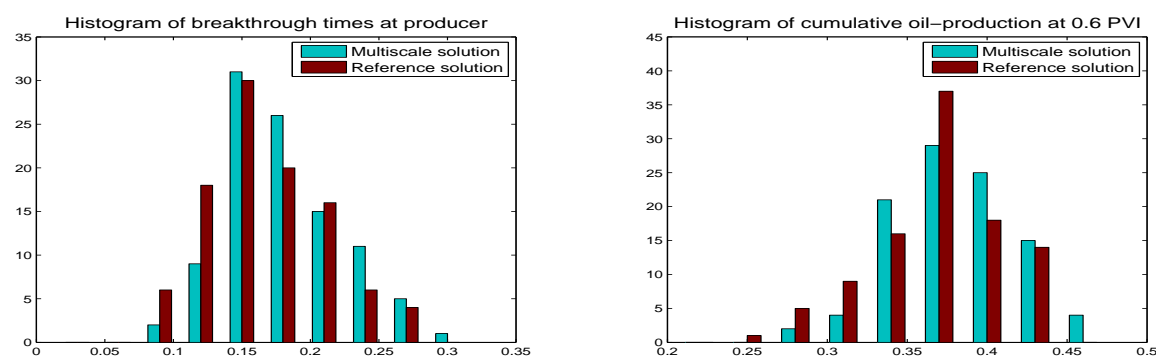

Figure 9: Histograms of the breakthrough times and cumulative oil production values shown in Figure 7. 

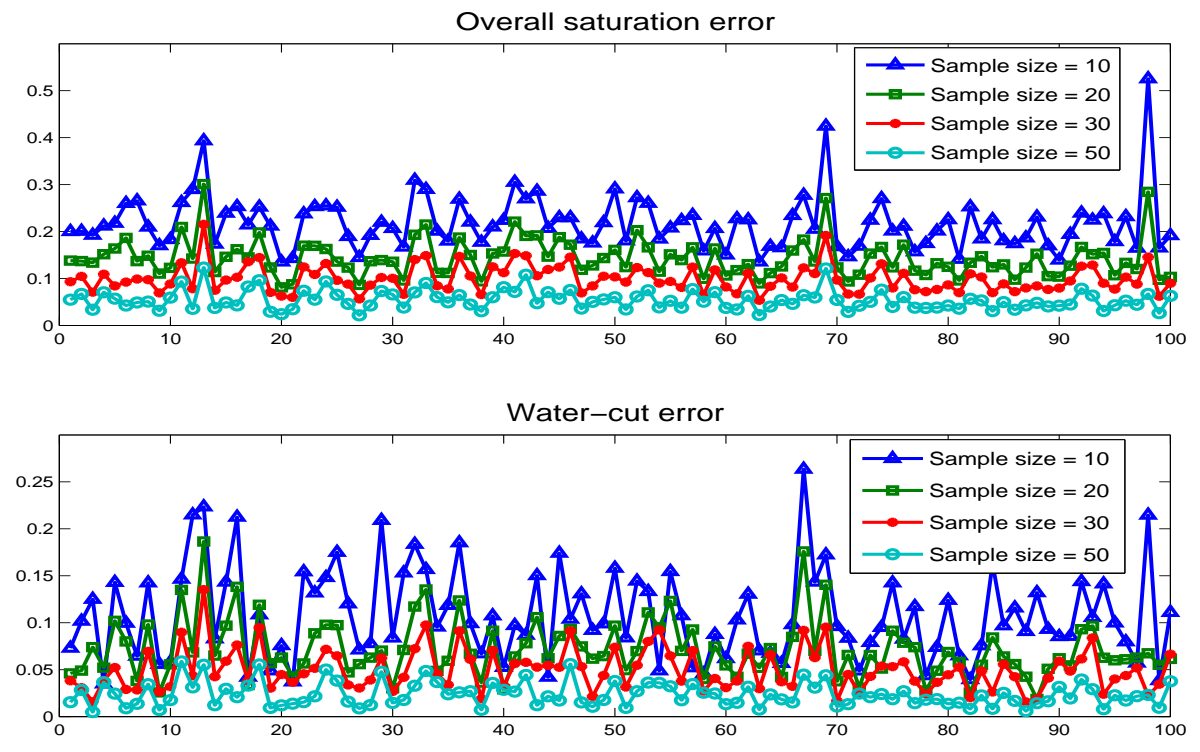

Figure 10: Saturation and water-cut error for solutions obtained using different number of permeability realizations to generate the multiscale basis functions.
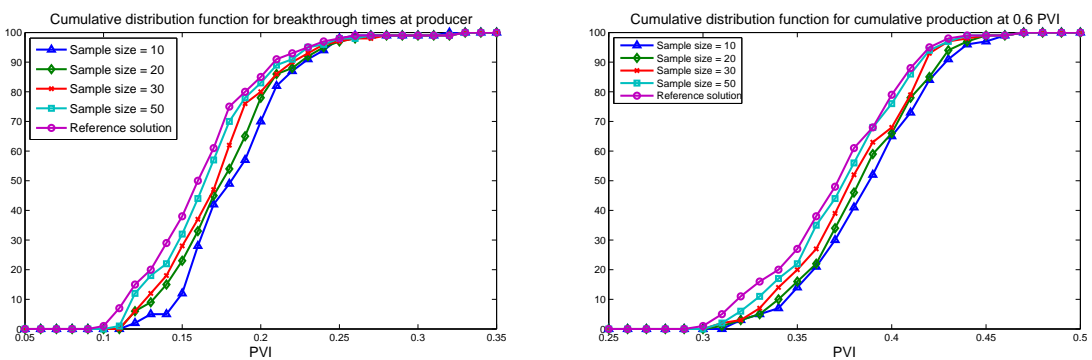

Figure 11: Cumulative probability distribution for breakthrough time and cumulative oil production at 0.6 PVI.
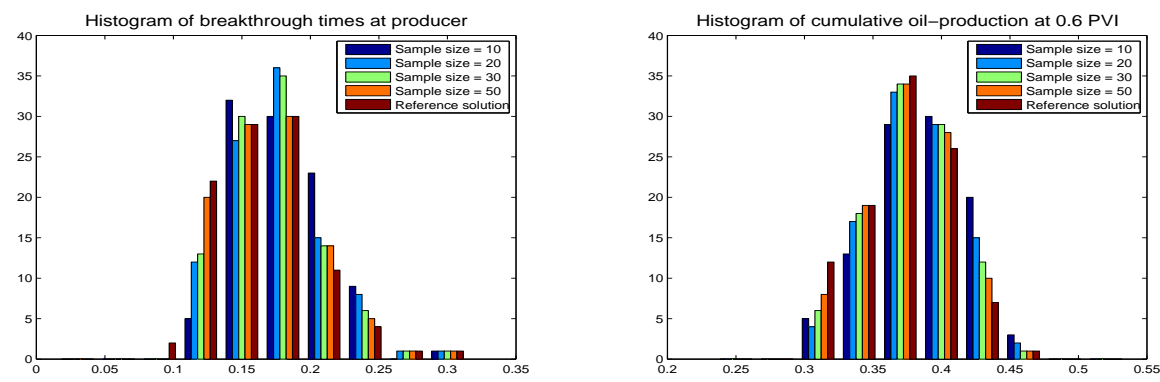

Figure 12: Histograms of the breakthrough times and cumulative oil production. 


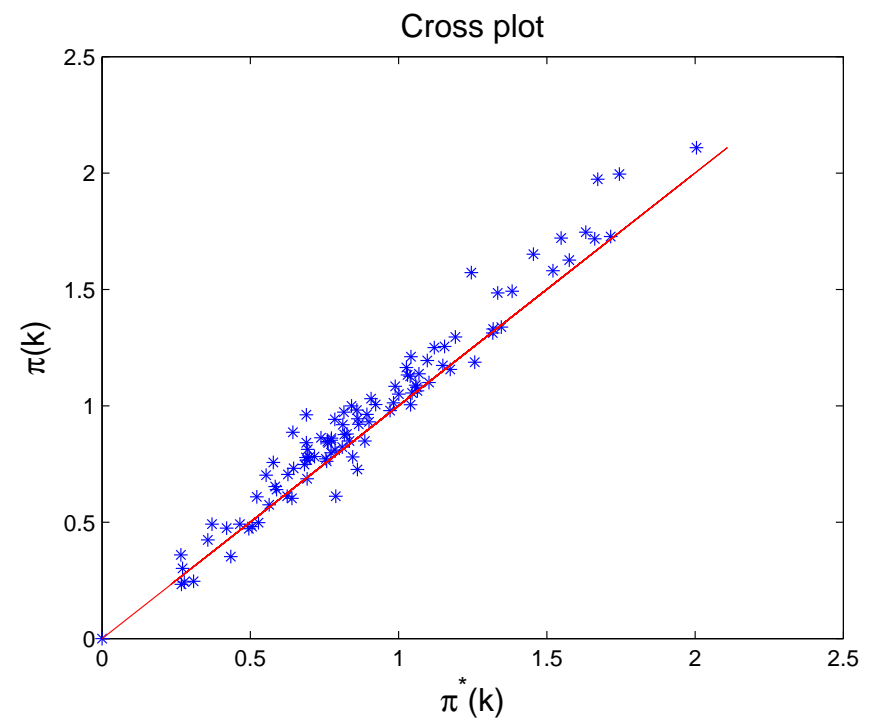

Figure 13: Cross plot between $\pi(k)$ and $\pi^{*}(k)$.

screen the fine-scale runs. Our main goal is to provide such inexpensive coarsescale runs that are statistically accurate. Our initial numerical results show that the proposed methods can be efficiently used in these application. One of the main criteria for these inexpensive simulations is strong correlation between $\pi(k)$ and $\pi^{*}(k)$, where $\pi^{*}(k)$ is approximate distribution. In Figure 13, we plot the cross plot of $\int_{0}^{T}\left(q_{w}(t)-q^{\text {obs }}(t)\right)^{2} d t$ for fine-scale and multiscale simulations. The strong correlation suggests that our approach can be successfully used in these uncertainty quantification problems. One can also test less expensive methods where the saturation equation is solved on the coarse grid or fast streamline techniques are used. These inexpensive computations are observed to be statistically accurate in $[20,16]$ when realization based multiscale finite element type methods are used. In future, we will study the stochastic mixed MsFEM combined with the upscaled transport equations.

\section{Conclusions}

In this paper, our goal is to develop mixed multiscale finite element methods (MsFEM) for solving stochastic flow equations described by second order elliptic equations with random coefficients. The proposed approaches compute multiscale basis functions on the coarse spatial grid. The pre-computed basis functions are constructed based on selected realizations of the stochastic permeability field, and thus span both spatial scales and uncertainties. These basis functions are used to solve the stochastic equation for an arbitrary realization on the coarse grid. 
We consider mixed formulation of the flow problem. The solution is projected onto the finite dimensional space spanned by pre-computed basis functions. The proposed method can be regarded as an extension of mixed MsFEM to stochastic porous media flow equations. We employ multiscale methods using limited global information since the permeability fields do not have apparent scale separation. The proposed approach does not require any interpolation in stochastic space and can accurately predict the solution on the coarse grid. In general, the uncertainty space can be partitioned into coarse regions where our approach can be used in each coarse patch.

We present numerical results for two-phase immiscible flow in stochastic porous media. In particular, we consider both Gaussian fields as well as permeability fields with exponential variogram. For the former case, due to smaller uncertainty space, we use lowest order interpolation points in uncertainty space for computing the basis functions. For the exponential case, due to large uncertainties, we employ randomly selected realizations for constructing basis functions. Numerical results show that for both cases, one can accurately predict the solutions with the multiscale method using only a few realizations to generate basis functions. It is particularly encouraging that the proposed multiscale methods are capable of predicting the stochastic solution with very few basis functions in high dimensional uncertainty space. We would like to note that the proposed approaches are not restricted to Cartesian grids and can be used for unstructured grids [4]. Finally, we would like to note that the proposed approaches can be easily combined with interpolation based approaches in order to achieve greater flexibility.

Although the results presented in this paper are encouraging, there is scope for further exploration. Our intent here was to demonstrate that one can capture the spatial and uncertainty variations of the stochastic flow solutions via precomputed basis functions. In particular, we would like to show that the method provides a foundation for fast simulations for a large number of realizations needed for the calculation of flow and transport statistics. Our initial numerical results show that the proposed approaches can be efficiently used to speed-up the uncertainty quantification problems of subsurface flows. Our future aim is to use the stochastic mixed MsFEM in preconditioning of Markov chain Monte Carlo simulations. In particular, we will study the statistical accuracy of the approaches where mixed MsFEM combined with both coarse-scale and fine-scale saturation solvers.

\section{Acknowledgment}

The research of J. A. is funded by the Research Council of Norway under grant no. 158908/I30 and by Shell and the Research Council of Norway under grant no. 175962/S30. The research of Y. E. is partially supported by NSF CMG/DMS grant 0621113 and DOE grant DE-FG02-05ER25669. We are grateful to Paul Dostert for performing initial numerical simulations on uncertainty quantification using the proposed mixed multiscale finite element method. 


\section{References}

[1] J. E. AARnEs, On the use of a mixed multiscale finite element method for greater flexibility and increased speed or improved accuracy in reservoir simulation, SIAM MMS, 2 (2004), pp. 421-439.

[2] J. E. Aarnes, Y. Efendiev, and L. Jiang, Analysis of multiscale finite element methods using global information for two-phase flow simulations, (submitted to Comp. Geo.)

[3] J. E. Aarnes, Y. Efendiev, And L. Jiang, Analysis of Multiscale Finite Element Methods using Global Information For Two-Phase Flow Simulations, submitted to SIAM MMS.

[4] J. E. Aarnes, S. Krogstad, And K.-A. Lie, A hierarchical multiscale method for two-phase flow based upon mixed finite elements and nonuniform grids, Multiscale Model. Simul. 5(2) (2006), pp. 337-363.

[5] J. E. Aarnes, S. Krogstad, And K.-A. Lie, Multiscale mixed/mimetic methods on corner-point grids, To appear in Comput. Geosci.

[6] T. Arbogast, Implementation of a locally conservative numerical subgrid upscaling scheme for two-phase Darcy flow, Comput. Geosci., 6 (2002), pp. 453-481. Locally conservative numerical methods for flow in porous media.

[7] T. Arbogast, G. Pencheva, M. F. Wheeler, and I. Yotov, A multiscale mortar mixed finite element method, SIAM J. Multiscale Modeling and Simulation, to appear.

[8] I. BabušKa, G. Caloz, And E. Osborn, Special finite element methods for a class of second order elliptic problems with rough coefficients, SIAM J. Numer. Anal., 31 (1994), pp. 945-981.

[9] I. BABušKa And E. Osborn, Generalized finite element methods: Their performance and their relation to mixed methods, SIAM J. Numer. Anal., 20 (1983), pp. 510-536.

[10] F. Brezzi, Interacting with the subgrid world, in Numerical analysis 1999 (Dundee), Chapman \& Hall/CRC, Boca Raton, FL, 2000, pp. 69-82.

[11] Y. Chen and L. Durlofsky, An ensemble level upscaling approach for efficient estimation of fine-scale production statistics using coarse-scale simulations, SPE paper 106086, presented at the SPE Reservoir Simulation Symposium, Houston, Feb. 26-28 (2007).

[12] Y. Chen, L. J. Durlofsky, M. Gerritsen, and X. H. Wen, A coupled local-global upscaling approach for simulating flow in highly heterogeneous formations, Advances in Water Resources, 26 (2003), pp. 1041-1060. 
[13] Z. Chen And T. Y. Hou, A mixed multiscale finite element method for elliptic problems with oscillating coefficients, Math. Comp., 72 (2002), pp. 541-576 (electronic).

[14] C. V. Deutsch And A. G. Journel, GSLIB: Geostatistical software library and user's guide, 2nd edition, Oxford University Press, New York, 1998.

[15] P. DosterT Uncertainty quantification using multiscale methods for porous media flows, Ph.D. thesis, Texas A\& M University, 2007.

[16] P. Dostert, Y. Efendiev, T. Hou, And W. Luo,, Coarse-gradient Langevin algorithms for dynamic data integration and uncertainty quantification, J. Comp. Physics, 217 (2006), pp. 123-142.

[17] W. E AND B. EngQuist, The heterogeneous multi-scale methods, Comm. Math. Sci., 1(1) (2003), pp. 87-133.

[18] Y. Efendiev, V. Ginting, T. Hou, And R. Ewing, Accurate multiscale finite element methods for two-phase flow simulations, J. Comp. Physics, 220 (1), pp. 155-174, 2006.

[19] Y. Efendiev, T. Hou, And V. Ginting, Multiscale finite element methods for nonlinear problems and their applications, Comm. Math. Sci., 2 (2004), pp. 553-589.

[20] Y. Efendiev, T. Hou And W. Luo, Preconditioning Markov chain Monte Carlo simulations using coarse-scale models, SIAM. Sci. Comp. 28(2), pp. 776-803, 2006.

[21] Y. Efendiev, T. Y. Hou, And X. H. Wu, Convergence of a nonconforming multiscale finite element method, SIAM J. Num. Anal., 37 (2000), pp. 888-910.

[22] T. Y. Hou AND X. H. Wu, A multiscale finite element method for elliptic problems in composite materials and porous media, Journal of Computational Physics, 134 (1997), pp. 169-189.

[23] T. Hughes, G. Feijoo, L. Mazzei, and J. Quincy, The variational multiscale method - a paradigm for computational mechanics, Comput. Methods Appl. Mech. Engrg, 166 (1998), pp. 3-24.

[24] P. Jenny, S. H. Lee, And H. TChelePI, Multi-scale finite volume method for elliptic problems in subsurface flow simulation, J. Comput. Phys., 187 (2003), pp. 47-67.

[25] M. LoÈve, Probability Theory, 4th ed., Springer, Berlin, 1977. 
[26] F. Nobile, R. Tempone, and C. G. Webster, A sparse grid stochastic collocation method for elliptic partial differential equations with random input data, MOX Technical Report 85, Dipartimento di Matematica, submitted to SIAM Journal of Numerical Analysis (2006).

[27] D. Oliver, L. Cunha, And A. Reynolds, Markov chain Monte Carlo methods for conditioning a permeability field to pressure data, Mathematical Geology, 29 (1997).

[28] H. Owhadi And L. Zhang, Metric based up-scaling, Comm. Pure Appl. Math., 2007.

[29] P. Raviart and J. Thomas, A mixed finite element method for second order elliptic equations, In: I. Galligani and E. Magenes (eds.): Mathematical Aspects of Finite Element Methods. Berlin - Heidelberg - New York: Springer-Verlag, pp. 292-315, 1977.

[30] C. Robert and G. Casella, Monte Carlo Statistical Methods, SpringerVerlag, New-York, 1999.

[31] E. Wong, Stochastic Processes in Information and Dynamical Systems, MCGraw-Hill, 1971.

[32] D. Xiu and J. Hesthaven, High-Order Collocation Methods for Differential Equations with Random Inputs, SIAM J. Sci. Comput. Wol. 27, No. 3, pp. 1118-1139. 